\title{
Self-similar solutions of a cross-diffusion parabolic system with variable density: explicit estimates and asymptotic behaviour
}

\author{
M. M. Aripov, A. S. Matyakubov \\ National University of Uzbekistan, Applied Mathematics and Computer Analysis, \\ Universitet, 4, Tashkent, 100174, Uzbekistan \\ mirsaidaripov@mail.ru, almasa@list.ru
}

PACS 02.30.Jr, 02.30.Mv, 11.10.Jj, 11.10.Lm

DOI 10.17586/2220-8054-2017-8-1-5-12

\begin{abstract}
In this paper, we study the properties of self-similar solutions of a cross-diffusion parabolic system. In particular, we find the ZeldovichBarenblatt type solution to the cross diffusive system. The asymptotic behavior of self-similar solutions are analyzed for both the slow and fast diffusive regimes. It is shown that coefficients of the main term of the asymptotic of solution satisfy some system of nonlinear algebraic equations.
\end{abstract}

Keywords: cross-diffusive system, non-divergence form, finite speed, perturbation, global solutions, asymptotic behavior, numerical analysis.

Received: 25 July 2016

Revised: 28 August 2016

\section{Introduction}

Consider in $Q=\left\{(t, x): t>0, x \in \mathbb{R}^{N}\right\}$ the cross-diffusive system of equations in non-divergence form with Cauchy conditions:

$$
\begin{gathered}
|x|^{n} \frac{\partial u}{\partial t}=v^{\alpha_{1}} \nabla\left(|x|^{k} u^{m_{1}-1} \nabla u\right)+|x|^{n} u^{\beta_{1}}, \\
|x|^{n} \frac{\partial v}{\partial t}=u^{\alpha_{2}} \nabla\left(|x|^{k} v^{m_{1}-1} \nabla v\right)+|x|^{n} v^{\beta_{2}}, \\
u(0, x)=u_{0}(x), \quad v(0, x)=v_{0}(x), x \in \mathbb{R}^{N} .
\end{gathered}
$$

where $n, k, m_{1}, m_{2}, \alpha_{1}, \alpha_{2}, \beta_{1}, \beta_{2}$ are the numerical parameters, $\nabla(\cdot)=\operatorname{grad}_{x}(\cdot)$, are the functions $u=$ $u(t, x) \geq 0, v=v(t, x) \geq 0$ are the solutions.

It is clear that the system (1) is degenerate. Therefore, it does not have classical solutions on the domain defined by equations $u(t, x)=0, v(t, x)=0, \nabla u=0, \nabla v=0$, meaning system (1) may not have a classical solution. Therefore, in this case we consider a weak solution having the property $u(t, x) \geq 0, v(t, x) \geq 0$; $v^{\alpha_{1}} \nabla\left(|x|^{k} u^{m_{1}-1} \nabla u\right), u^{\alpha_{2}} \nabla\left(|x|^{k} v^{m_{1}-1} \nabla v\right) \in C(Q)$ and obeying to the system (1) in sense of a distribution [1].

Non-divergent form equations and system of equations (1) are often used to describe various physical phenomena, such as the diffusive process for biological species, the resistive diffusion phenomena in force-free magnetic fields, curve shortening flow, spreading of infectious disease and so on, see for [2-8].

In [2] for the system (1) (for $n=0, k=0$, and $m_{1}=1, m_{2}=1$ ) the authors proved that: (i) when $\min \{a, b\} \leq \lambda_{1}$, then there exists global positive classical solution, and all positive classical solutions cannot blow up in finite time in the meaning of maximum norm; (ii) when $\min \{a, b\}>\lambda_{1}$, there is no global positive classical solution.

Previous studies [3] with positive solutions of some degenerate and quasilinear parabolic systems are given by:

$$
\begin{aligned}
& u_{i t}=f_{i}\left(u_{i+1}\right)\left(\Delta u_{i}+a_{i} u_{i}\right) \\
& u_{n t}=f_{n}\left(u_{1}\right)\left(\Delta u_{n}+a_{n} u_{n}\right), \quad x \in \Omega, t>0, i=1,2, \ldots, n-1 .
\end{aligned}
$$

The local existence and uniqueness of classical solution also proven in [3]. It is also shown that: (i) when $\min \left\{a_{1}, \ldots, a_{n}\right\} \leq \lambda_{1}$ then there exists global positive classical solution, and all positive classical solutions cannot blow up in finite time in the meaning of maximum norm; (ii) when $\min \left\{a_{1}, \ldots, a_{n}\right\}>\lambda_{1}$, and the initial datum $\left(u_{10}, \ldots, u_{n 0}\right)$ satisfies some assumptions, then the positive classical solution is unique and blows up in finite time, where $\lambda_{1}$ is the first eigenvalue of $\Delta$ in $\Omega$ with homogeneous Dirichlet boundary conditions.

Some properties of solutions for the non-divergence form in single equation, such as the existence, nonuniqueness, the blow-up properties etc. have been discussed by many authors [4-6]. Finite speed properties of a 
perturbation of distribution (FSPD) and the asymptotic behavior of self-similar solutions for another systems are considered in [9-12].

In $[13,14]$, the Cauchy problem for the following two equations with variable coefficients is studied:

$$
\rho(x) \frac{\partial u}{\partial t}=\operatorname{div}\left(u^{m-1}|\nabla u|^{p-2} \nabla u\right)+\rho(x) u^{\beta},
$$

and

$$
\rho(x) \frac{\partial u}{\partial t}=\operatorname{div}\left(u^{m-1}|\nabla u|^{p-2} \nabla u\right)+u^{\beta}, x \in \mathbb{R}^{N}, t>0,
$$

where $p>1, m+p-3>0, \beta>m+p-2, \rho(x)=|x|^{-n}$ or $\rho(x)=(1+|x|)^{-n}$. The authors showed that under some constraints for the parameters and for the initial data, any nontrivial solution of the Cauchy problem blows up in finite time. Moreover, the authors established a sharp universal estimate of the solution near the blow-up point.

Ref. [7] considers the degenerate and quasilinear parabolic systems having non divergent form given by:

$$
\left\{\begin{array}{l}
u_{i t}=u_{i}^{p_{i}}\left(\Delta u_{i}+a_{i} u_{i+1}\right), i=1,2, \ldots, m, u_{m+1}=u_{1}, \\
u_{i}(x, 0)=u_{i 0}(x), i=1,2, \ldots, m, x \in \Omega, \\
u_{i}(x, t)=0, i=1,2, \ldots, m, x \in \partial \Omega, t>0,
\end{array}\right.
$$

where $a_{i}, p_{i}$ are the positive constants, $\Omega \in \mathbb{R}^{N}$ is a bounded domain with smooth boundary $\partial \Omega$. The local existence and uniqueness of classical solution are proved. Moreover, it proved that all solutions exist globally with homogeneous Dirichlet boundary conditions.

Ref. [8], investigates positive solutions of the degenerate parabolic equation not in divergence form: $u_{t}=$ $u^{p} \Delta u+u^{q}|\nabla u|^{2}-u^{r}$, subject to null Dirichlet boundary conditions. The existence of global solutions and the large time behavior for them is studied. The main effort is paid to obtain uniform asymptotic profiles for decay solutions, under various dominations of the nonlinear diffusion or absorption. It is shown that the large time property of the solution $u$ behaves just like $(1+(r-1) t)(-1 / r-1)$ if the decay is governed by the nonlinear absorption with $1<r<p+1$.

In this paper we consider the problem of constructing Zeldovich-Barenblatt type solution for the cross system equation (1). Using comparison methods, the property of FSPD of the Cauchy problem for a cross-diffusion parabolic system not in divergence form is established. The asymptotic behavior is discussed for a solution of the cross-diffusion parabolic system equations in non-divergence form for slow and fast diffusion cases depending on the value of the numerical parameters. On the basis of the asymptotic of solutions, suitable initial approximations are offered for the iterative process in the cases of the slow and fast diffusions, depending on the numerical parameter values.

\section{The self-similar system of equations}

Here, we provide a method of nonlinear splitting [15] for construction of self-similar equation for the system given by Eqs. (1). We look for the solutions $u(t, x), v(t, x)$ in the form:

$$
\begin{aligned}
& u(t, x)=\bar{u}(t) w(\tau(t), r), \\
& v(t, x)=\bar{v}(t) \psi(\tau(t), r) .
\end{aligned}
$$

Then, we obtain $\bar{u}(t), \bar{v}(t)$ as $\bar{u}(t)=(T+t)^{1 /\left(1-\beta_{1}\right)}, \bar{v}(t)=(T+t)^{1 /\left(1-\beta_{2}\right)}$.

From Eqs. (3) and (1), we obtain the following system of equations:

$$
\begin{aligned}
& r^{n} \frac{\partial w}{\partial \tau}=\psi^{\alpha_{1}} r^{1-N} \frac{\partial}{\partial r}\left(r^{N-1+k} w^{m_{1}-1} \frac{\partial w}{\partial r}\right)+b_{1} r^{n} \tau^{-1}\left(w^{\beta_{1}}-\frac{1}{1-\beta_{1}} w\right), \\
& r^{n} \frac{\partial \psi}{\partial \tau}=w^{\alpha_{2}} r^{1-N} \frac{\partial}{\partial r}\left(r^{N-1+k} \psi^{m_{2}-1} \frac{\partial \psi}{\partial r}\right)+b_{2} r^{n} \tau^{-1}\left(\psi^{\beta_{2}}-\frac{1}{1-\beta_{2}} \psi\right),
\end{aligned}
$$


where:

$$
\begin{aligned}
& \tau(t)=\int(T+t)^{\frac{\alpha_{1}}{1-\beta_{2}}+\frac{m_{1}-1}{1-\beta_{1}}} d t=\int(T+t)^{\frac{\alpha_{2}}{1-\beta_{1}}+\frac{m_{2}-1}{1-\beta_{2}}} d t \quad \text { at } \quad \frac{\alpha_{1}}{1-\beta_{2}}+\frac{m_{1}-1}{1-\beta_{1}}+1 \neq 0, \\
& \tau(t)=\ln (T+t) \quad \text { at } \quad \frac{\alpha_{1}}{1-\beta_{2}}+\frac{m_{1}-1}{1-\beta_{1}}+1=0, \\
& b_{i}=\left(\frac{\alpha_{i}}{1-\beta_{3-i}}+\frac{m_{i}-1}{1-\beta_{i}}+1\right)^{-1}, i=1,2, r=|x|, \\
& \frac{\alpha_{1}}{1-\beta_{2}}+\frac{m_{1}-1}{1-\beta_{1}}=\frac{\alpha_{2}}{1-\beta_{1}}+\frac{m_{2}-1}{1-\beta_{2}} .
\end{aligned}
$$

It is easy to show that the system (4) has approximately self-similar solution given by:

$$
w(\tau, r)=f(\xi), \quad \psi(\tau, r)=\phi(\xi), \quad \xi=r^{\frac{2-k}{2}} / \tau^{\frac{2-k}{4-2 k+2 n}},
$$

where $\xi$ is self-similar variable and the functions $f(\xi), \phi(\xi)$ fulfill the following approximately self-similar system of equations:

$$
\begin{aligned}
& \phi^{\alpha_{1}} \xi^{1-\frac{2 N}{2-k}} \frac{d}{d \xi}\left(\xi^{\frac{2 N}{2-k}-1} f^{m_{1}-1} \frac{d f}{d \xi}\right)+\frac{2}{(2-k)(n+2-k)} \xi^{\frac{2 N}{2-k}+1} \frac{d f}{d \xi}+\frac{4 b_{1}}{(2-k)^{2}} \xi^{\frac{2 N}{2-k}}\left(f^{\beta_{1}}-\frac{1}{1-\beta_{1}} f\right)=0 \\
& f^{\alpha_{2}} \xi^{1-\frac{2 N}{2-k}} \frac{d}{d \xi}\left(\xi^{\frac{2 N}{2-k}-1} \phi^{m_{2}-1} \frac{d \phi}{d \xi}\right)+\frac{2}{(2-k)(n+2-k)} \xi^{\frac{2 N}{2-k}+1} \frac{d \phi}{d \xi}+\frac{4 b_{2}}{(2-k)^{2}} \xi^{\frac{2 N}{2-k}}\left(\phi^{\beta_{2}}-\frac{1}{1-\beta_{2}} \phi\right)=0 .
\end{aligned}
$$

In the following, we will consider nontrivial, nonnegative solutions of the system (6) satisfying the following conditions:

$$
\begin{aligned}
& f(0)=M_{1}, \phi(0)=M_{2}, M_{1} \in \mathbb{R}^{+}, M_{2} \in \mathbb{R}^{+}, \\
& f\left(d_{1}\right)=\phi\left(d_{2}\right)=0,0<d_{1}<\infty, \quad 0<d_{2}<\infty .
\end{aligned}
$$

\section{The slow diffusion case $\left(m_{3-i}>1+\alpha_{i}, i=1,2\right)$. Explicit estimates of the solution of problem (1)-(2)}

Using the solution comparison method of [1] and the standard equations method of [15] for solving the problem (6)-(7), we can obtain the estimates for the solution of the problem (1)-(2).

We note that the functions:

$$
\bar{f}(\xi)=A\left(a-\xi^{\frac{2 n}{2-k}+2}\right)_{+}^{p_{1}}, \quad \bar{\phi}(\xi)=B\left(a-\xi^{\frac{2 n}{2-k}+2}\right)_{+}^{p_{2}},
$$

where $p_{i}=\frac{m_{3-i}-\alpha_{i}-1}{\left(m_{1}-1\right)\left(m_{2}-1\right)-\alpha_{1} \alpha_{2}}, i=1,2,(b)_{+}=\max (0, b), a^{p_{1}} A=M_{1}, a^{p_{2}} B=M_{2}$ at $\xi<a^{\left(\frac{2 n}{2-k}+2\right)^{-1}}$ satisfy Eq. (7).

Theorem 1. Let $m_{3-i}>1+\alpha_{i}, p_{i} m_{i}-1>0, i=1,2$,

$$
\begin{gathered}
M_{i}^{\beta_{i}-1} \leq \frac{1}{1-\beta_{i}}+\frac{N+n}{n+2-k} \frac{p_{i}}{b_{i}\left(p_{i} m_{i}-1\right)}, \quad i=1,2, \\
M_{i}^{m_{i}-1} M_{3-i}^{\alpha_{i}}=\frac{a}{\left(p_{i} m_{i}-1\right)(n+2-k)^{2}}, \quad i=1,2, \\
u_{+}(0, x) \geq u_{0}(x), \quad v_{+}(0, x) \geq v_{0}(x), \quad x \in \mathbb{R}^{N} .
\end{gathered}
$$

Then, in $Q$ the problem (1)-(2) has a global solution with the estimate:

$$
\begin{aligned}
& u(t, x) \leq u_{+}(t, x)=\bar{u}(t) \bar{f}(\xi), \\
& v(t, x) \leq v_{+}(t, x)=\bar{v}(t) \bar{\phi}(\xi) .
\end{aligned}
$$

Proof. Theorem 1 is proved by the method of comparison of solutions. As comparable functions, we take the functions $u_{+}(t, x), v_{+}(t, x)$, defined by Eq. (9). Then, according to (3), (5) and $M_{i}^{m_{i}-1} M_{3-i}^{\alpha_{i}}=\frac{a}{\left(p_{i} m_{i}-1\right)(n+2-k)^{2}}$, $i=1,2$, we get:

$$
\begin{aligned}
L u_{+}(t, x) & =\left(-\frac{4 p_{1}(N+n)}{(2-k)^{2}(n+2-k)\left(p_{1} m_{1}-1\right)}-\frac{4 b_{1}}{\left(1-\beta_{1}\right)(2-k)^{2}}\right) \xi^{\frac{2 n}{2-k}} \bar{f}(\xi)+\frac{4 b_{1}}{(2-k)^{2}} \xi^{\frac{2 n}{2-k}} \bar{f}^{\beta_{1}}(\xi), \\
L v_{+}(t, x) & =\left(-\frac{4 p_{2}(N+n)}{(2-k)^{2}(n+2-k)\left(p_{2} m_{2}-1\right)}-\frac{4 b_{2}}{\left(1-\beta_{2}\right)(2-k)^{2}}\right) \xi^{\frac{2 n}{2-k}} \bar{\phi}(\xi)+\frac{4 b_{2}}{(2-k)^{2}} \xi^{\frac{2 n}{2-k}} \bar{\phi}^{\beta_{2}}(\xi) .
\end{aligned}
$$


From these expressions and $\bar{f}(\xi) \leq A a^{p_{1}}=M_{1}, \bar{\phi}(\xi) \leq B a^{p_{2}}=M_{2}$, we find, that for execution, the inequalities $L u_{+} \leq 0, L v_{+} \leq 0$ are enough to fulfill the conditions

$$
M_{i}^{\beta_{i}-1} \leq \frac{1}{1-\beta_{i}}+\frac{N+n}{n+2-k} \frac{p_{i}}{b_{i}\left(p_{i} m_{i}-1\right)}, \quad i=1,2 .
$$

By the conditions of the theorem, the last inequalities are satisfied. Then, according to the theorem, for comparison of problem (1)-(2) solutions, there is a global solution in $Q$ and the following estimation $u_{+}(t, x) \geq$ $u(t, x), v_{+}(t, x) \geq v(t, x), x \in \mathbb{R}^{N}$ holds.

We note for the solutions of Cauchy problem (1)-(2), we have FSPD properties.

\section{The asymptotic behavior of self-similar solutions of the problem (6)-(7)}

Let us introduce the following notations:

$$
b_{i 1}=p_{i}\left(p_{i} m_{i}-1\right), b_{i 2}=-\frac{p_{i}}{(n+2-k)^{2}}, b_{i 3}=\frac{b_{i}}{(n+2-k)^{2} a}, \quad i=1,2 .
$$

Assume $\frac{\alpha_{1}}{1-\beta_{2}}+\frac{m_{1}-1}{1-\beta_{1}}=\frac{\alpha_{2}}{1-\beta_{1}}+\frac{m_{2}-1}{1-\beta_{2}}$. Then the following theorem is valid:

Theorem 2. Let $\frac{\alpha_{1}}{1-\beta_{2}}+\frac{m_{1}-1}{1-\beta_{1}}+1>0$. Then compactly supported solution of the problem (6), (7) as $\xi \rightarrow a^{\left(\frac{2 n}{2-k}+2\right)^{-1}}$ has the following asymptotic behavior:

$$
\begin{aligned}
& f(\xi)=c_{1}\left(a-\xi^{\frac{2 n}{2-k}+2}\right)^{p_{1}}(1+o(1)), \\
& \phi(\xi)=c_{2}\left(a-\xi^{\frac{2 n}{2-k}+2}\right)^{p_{2}}(1+o(1)),
\end{aligned}
$$

if one of the the following conditions are fulfilled:

(1) $p_{1}=\frac{1}{1-\beta_{1}}, p_{2}=\frac{1}{1-\beta_{2}}$ and the coefficients $c_{i}(i=1,2)$ are the roots of the systems of nonlinear algebraic equations:

$$
b_{i 1} c_{i}^{m_{i}-1} c_{3-i}^{\alpha_{i}}+b_{i 2}+b_{i 3} c_{i}^{\beta_{i}-1}=0, \quad i=1,2
$$

(2) $p_{i}<\frac{1}{1-\beta_{i}}, p_{i} m_{i}>1, i=1,2$ and the coefficients $c_{i}(i=1,2)$ are the roots of the systems of nonlinear algebraic equations:

$$
b_{i 1} c_{i}^{m_{i}-1} c_{3-i}^{\alpha_{i}}+b_{i 2}=0, \quad i=1,2
$$

(3) $p_{1}=\frac{1}{1-\beta_{1}}, p_{2}<\frac{1}{1-\beta_{2}}, p_{2} m_{2}>1$ and the coefficients $c_{i}(i=1,2)$ are the roots of the systems of nonlinear algebraic equations:

$$
\left\{\begin{array}{l}
b_{11} c_{1}^{m_{1}-1} c_{2}^{\alpha_{1}}+b_{12}+b_{13} c_{1}^{\beta_{1}-1}=0 \\
b_{21} c_{2}^{m_{2}-1} c_{1}^{\alpha_{2}}+b_{22}=0
\end{array}\right.
$$

(4) $p_{1}<\frac{1}{1-\beta_{1}}, p_{2}=\frac{1}{1-\beta_{2}}, p_{1} m_{1}>1$ and the coefficients $c_{i}(i=1,2)$ are the roots of the systems of nonlinear algebraic equations:

$$
\left\{\begin{array}{l}
b_{11} c_{1}^{m_{1}-1} c_{2}^{\alpha_{1}}+b_{12}=0 \\
b_{21} c_{2}^{m_{2}-1} c_{1}^{\alpha_{2}}+b_{22}+b_{23} c_{2}^{\beta_{2}-1}=0
\end{array}\right.
$$

Proof. To simplify such auxiliary systems of equations, one can use the following transformations:

$$
f(\xi)=\bar{f}(\xi) y_{1}(\eta), \phi(\xi)=\bar{\phi}(\xi) y_{2}(\eta), \eta=-\ln \left(a-\xi^{\frac{2 n}{2-k}+2}\right),
$$

where $\bar{f}(\xi)=\left(a-\xi^{\frac{2 n}{2-k}+2}\right)^{p_{1}}, \bar{\phi}(\xi)=\left(a-\xi^{\frac{2 n}{2-k}+2}\right)^{p_{2}}$ and $y_{1}(\eta), y_{2}(\eta)-$ the new functions.

Now, let us study the asymptotic behavior of solutions of Eqs. (6) and (7) at $\xi \rightarrow a^{\left(\frac{2 n}{2-k}+2\right)^{-1}}$.

After transformation (11), Eqs. (6) and (7) will take the following form:

$$
y_{3-i}^{\alpha_{i}} \frac{d}{d \eta}\left(L y_{i}\right)+a_{i 1}(\eta) y_{3-i}^{\alpha_{i}} L y_{i}+a_{i 2}(\eta)\left(\frac{d y_{i}}{d \eta}+a_{i 0}(\eta) y_{i}\right)+a_{i 3}(\eta) y_{i}+a_{i 4}(\eta) y_{i}^{\beta_{i}}=0,(i=1,2)
$$


Here,

$$
\begin{gathered}
L y_{i}=y_{i}^{m_{i}-1}\left(\frac{d y_{i}}{d \eta}+a_{i 0}(\eta) y_{i}\right), \quad a_{i 0}(\eta)=-p_{i}, \quad a_{i 1}(\eta)=\frac{n+N}{n+2-k} \frac{e^{-\eta}}{a-e^{-\eta}}-p_{i} m_{i}+1, \\
a_{i 2}(\eta)=\frac{1}{(n+2-k)^{2}}, \quad a_{i 3}(\eta)=-\frac{b_{i}}{(n+2-k)^{2}\left(1-\beta_{i}\right)} \frac{e^{-\eta}}{a-e^{-\eta}}, \quad a_{i 4}(\eta)=\frac{b_{i}}{(n+2-k)^{2}} \frac{e^{-\left(1+p_{i}\left(\beta_{i}-1\right)\right) \eta}}{a-e^{-\eta}},
\end{gathered}
$$

We assumed that $\xi \in\left[\xi_{0}, \xi_{1}\right), 0<\xi_{0}<\xi_{1}, \xi_{1}=a^{\left(\frac{2 n}{2-k}+2\right)^{-1}}$. Therefore, the function $\eta(\xi)$ has the following properties:

$$
\eta^{\prime}(\xi)>0 \text { at } \xi \in\left[\xi_{0}, \xi_{1}\right), \quad \eta_{0}=\eta\left(\xi_{0}\right)>0, \quad \lim _{\xi \rightarrow \xi_{1}} \eta(\xi)=+\infty .
$$

The auxiliary system of equations (12) studied under the following limitations:

$$
\lim _{\eta \rightarrow+\infty} a_{i j}(\eta)=a_{i j}^{0} \quad(i=1,2 ; j=0,1,2,3,4),
$$

exist and are finite and nonzero, that is $0<\left|a_{i j}^{0}\right|<+\infty$.

The solutions of the system (12), in a certain vicinity of $+\infty$ satisfy the inequalities:

$$
y_{i}(\eta)>0, \quad y_{i}^{\prime}+a_{i 0}(\eta) y_{i} \neq 0 \quad(i=1,2) .
$$

Assuming in Eqs. (12):

we obtain the identity:

$$
v_{i}(\eta)=y_{i}^{m_{i}-1}\left(\frac{d y_{i}}{d \eta}+a_{i 0}(\eta) y_{i}\right), \quad i=1,2
$$

$$
v_{i}^{\prime}(\eta) \equiv-a_{i 1}(\eta) v_{i}(\eta)-a_{i 2}(\eta) v_{i}(\eta) y_{i}^{1-m_{i}} y_{3-i}^{-\alpha_{i}}-a_{i 3}(\eta) y_{i} y_{3-i}^{-\alpha_{i}}-a_{i 4}(\eta) y_{i}^{\beta_{i}} y_{3-i}^{-\alpha_{i}}, \quad(i=1,2) .
$$

Furthermore, consider the functions:

$$
g_{i}\left(\lambda_{i}, \eta\right) \equiv-a_{i 1}(\eta) \lambda_{i}-a_{i 2}(\eta) \lambda_{i} y_{i}^{1-m_{i}} y_{3-i}^{-\alpha_{i}}-a_{i 3}(\eta) y_{i} y_{3-i}^{-\alpha_{i}}-a_{i 4}(\eta) y_{i}^{\beta_{i}} y_{3-i}^{-\alpha_{i}},
$$

where $\lambda_{i} \in \mathbb{R},(i=1,2)$.

Let us suppose $\frac{\alpha_{1}}{1-\beta_{2}}+\frac{m_{1}-1}{1-\beta_{1}}+1>0$. Then,

$$
\begin{gathered}
\lim _{\eta \rightarrow+\infty} a_{i 1}(\eta)=-p_{i} m_{i}+1, \quad \lim _{\eta \rightarrow+\infty} a_{i 3}(\eta)=0, \quad \lim _{\eta \rightarrow+\infty} a_{i 2}(\eta)=\frac{1}{(n+2-k)^{2}}, \\
\lim _{\eta \rightarrow+\infty} a_{i 4}(\eta)= \begin{cases}\frac{b_{i}}{a(n+2-k)^{2}}, & \text { at } 1+p_{i}\left(\beta_{i}-1\right)=0, \\
0, & \text { at } 1+p_{i}\left(\beta_{i}-1\right)>0, \quad i=1,2 .\end{cases}
\end{gathered}
$$

and the functions $g_{i}\left(\lambda_{i}, \eta\right)(i=1,2)$ preserve sign on some interval $\left[\eta_{1},+\infty\right) \subset\left[\eta_{0},+\infty\right)$ for every fixed value $\lambda_{i}$ $(i=1,2)$.

Therefore, the functions $g_{i}\left(\lambda_{i}, \eta\right)(i=1,2)$ for all $\eta \in\left[\eta_{1},+\infty\right)$ satisfies one of the inequalities:

$$
g_{i}\left(\lambda_{i}, \eta\right)>0 \quad \text { or } \quad g_{i}\left(\lambda_{i}, \eta\right)<0 \quad(i=1,2) .
$$

Let us assume that for the functions $v_{i}(\eta)(i=1,2)$ the limit at $\eta \rightarrow+\infty$ does not exist. Consider the case, where one of the inequalities (16) are fulfilled. By force of the function's $v_{i}(\eta)(i=1,2)$ oscillation, the straight line $\bar{v}_{i}=\lambda_{i}(i=1,2)$ and its graph intersects an infinite number of times in the interval $\left[\eta_{i},+\infty\right)(i=1,2)$. However, this is impossible, since the interval $\left[\eta_{i},+\infty\right)(i=1,2)$ is justly one of the inequalities (16) and therefore, it follows from Eq. (15), that graph of the functions $v_{i}(\eta)(i=1,2)$ intersects the straight line $\bar{v}_{i}=\lambda_{i}(i=1,2)$ only once in the interval $\left[\eta_{i},+\infty\right)(i=1,2)$. Therefore, for the functions $v_{i}(\eta)(i=1,2)$ the limit at $\eta \rightarrow+\infty$ exists.

Assuming the functions $v_{i}(\eta)(i=1,2)$ are defined in accordance with Eq. (13) and have a limit at $\eta \rightarrow+\infty$, one can show that $y_{i}^{\prime}(\eta)(i=1,2)$ has a limit at $\eta \rightarrow+\infty$, which is equal to zero. Then,

$$
v_{i}(\eta)=y_{i}^{m_{i}-1}\left(\frac{d y_{i}}{d \eta}+a_{i 0}(\eta) y_{i}\right)=a_{i 0}^{0}\left(y_{i}^{0}\right)^{m_{i}}+o(1), i=1,2 \text { at } \eta \rightarrow+\infty
$$

and by (14) derivative of functions $v_{i}(\eta)(i=1,2)$ has a limit at $\eta \rightarrow+\infty$, which obviously equals zero.

Consequently,

$$
\lim _{\eta \rightarrow+\infty}\left(a_{i 1}(\eta) v_{i}(\eta)+a_{i 2}(\eta) v_{i}(\eta) y_{i}^{1-m_{i}} y_{3-i}^{-\alpha_{i}}+a_{i 3}(\eta) y_{i} y_{3-i}^{-\alpha_{i}}+a_{i 4}(\eta) y_{i}^{\beta_{i}} y_{3-i}^{-\alpha_{i}}\right)=0 \quad(i=1,2) .
$$


It is easy to see that the system (12) has a solution $\left(y_{1}(\eta), y_{2}(\eta)\right)$ with a finite non-zero limit at $\eta \rightarrow+\infty$ necessary to comply with the conditions of Theorem 2 . Then the compactly supported solution of the problem (6)-(7) has an asymptotic of the form (10) as $\xi \rightarrow a^{\left(\frac{2 n}{2-k}+2\right)^{-1}}$.

Thus, the theorem is proved.

Corollary 1. A weak solution of the problem (1)-(2) has the following asymptotic form:

$$
\begin{aligned}
& u_{A}(t, x) \approx c_{1}(T+t)^{\frac{1}{1-\beta_{1}}}\left(a-\left(\frac{|x|^{\frac{2-k}{2}}}{\tau^{\left(\frac{2 n}{2-k}+2\right)^{-1}}}\right)^{\frac{2 n}{2-k}+2}\right)^{p_{1}}, \\
& v_{A}(t, x) \approx c_{2}(T+t)^{\frac{1}{1-\beta_{2}}}\left(a-\left(\frac{|x|^{\frac{2-k}{2}}}{\tau^{\left(\frac{2 n}{2-k}+2\right)^{-1}}}\right)^{\frac{2 n}{2-k}+2}\right)^{p_{2}},
\end{aligned}
$$

at $|x| \rightarrow a^{\frac{1}{2-k+n}} \tau^{\frac{1}{n+2-k}}$, where constants $c_{1}, c_{2}, p_{1}, p_{2}$ are defined above.

5. Fast diffusion case $\left(p_{i}<0, i=1,2\right)$. Explicit estimates of the solution of problem (1)-(2)

Suppose that for system of equations (6) the following conditions are fulfilled:

$$
f^{\prime}(0)=0, \phi^{\prime}(0)=0, f(\infty)=0, \phi(\infty)=0 .
$$

Then, the functions

$$
\bar{f}(\xi)=A_{1}\left(a+\xi^{\frac{2 n}{2-k}+2}\right)^{p_{1}}, \quad \bar{\phi}(\xi)=A_{2}\left(a+\xi^{\frac{2 n}{2-k}+2}\right)^{p_{2}}, \quad p_{i}=\frac{m_{3-i}-1-\alpha_{i}}{\left(m_{1}-1\right)\left(m_{2}-1\right)-\alpha_{1} \alpha_{2}}, \quad i=1,2,
$$

satisfy Eq. (17), where $a>0$.

Theorem 3. Let $p_{i}<0, \beta_{i}<1, \frac{p_{i}}{1-p_{i} m_{i}} \frac{n+N}{n+2-k}-\frac{b_{i}}{1-\beta_{i}}+b_{i} a^{p_{i}\left(\beta_{i}-1\right)} \geq 0$, $A_{i}^{m_{i}-1} A_{3-i}^{\alpha_{i}}=\frac{1}{\left(1-p_{i} m_{i}\right)(n+2-k)^{2}}, i=1,2, u_{0}(x) \geq u_{-}(0, x), v_{0}(x) \geq v_{-}(0, x), x \in \mathbb{R}^{N}$.

Then, for problem (1)-(2), there is a global solution in $Q$ and for it, the following estimation is correct: $u(t, x) \geq u_{-}(t, x), v(t, x) \geq v_{-}(t, x), x \in \mathbb{R}$, where

$$
u_{-}(t, x)=\bar{u}(t) \bar{f}(\xi), \quad v_{-}(t, x)=\bar{v}(t) \bar{\phi}(\xi) .
$$

Theorem 3 is proved by the solution comparison method similar to the proof of Theorem 1 .

Furthermore, we introduce the following notations:

$$
b_{i 4}=p_{i}\left(\frac{n+N}{n+2-k}+p_{i} m_{i}-1\right), \quad b_{i 5}=\frac{p_{i}}{(n+2-k)^{2}}-\frac{b_{i}}{1-\beta_{i}(n+2-k)^{2}}, \quad i=1,2 .
$$

Also, we assume $\frac{\alpha_{1}}{1-\beta_{2}}+\frac{m_{1}-1}{1-\beta_{1}}=\frac{\alpha_{2}}{1-\beta_{1}}+\frac{m_{2}-1}{1-\beta_{2}}$. Then the following theorem is valid:

Theorem 4. Let $\frac{\alpha_{1}}{1-\beta_{2}}+\frac{m_{1}-1}{1-\beta_{1}}+1>0, \beta_{i}>1, p_{i}<0, i=1,2$. Then, vanishing at infinity, the solution of the problem (6), (17) at $\xi \rightarrow \infty$ has an asymptotic:

$$
f(\xi)=c_{3}\left(a+\xi^{\frac{2 n}{2-k}+2}\right)^{p_{1}}, \quad \phi(\xi)=c_{4}\left(a+\xi^{\frac{2 n}{2-k}+2}\right)^{p_{2}},
$$

where the coefficients $c_{i}(i=3,4)$ are the roots of systems of the nonlinear algebraic equations:

$$
b_{i 4} c_{i+2}^{m_{i}-1} c_{5-i}^{\alpha_{i}}+b_{i 5}=0, i=1,2 .
$$

The proof of the Theorem 4 is similar to that of the Theorem 2.

Corollary 2. A weak solution of the problem (1)-(2) has the following asymptotic:

$$
\begin{aligned}
& u_{A}(t, x) \approx c_{3}(T+t)^{\frac{1}{1-\beta_{1}}}\left(a+\left(\frac{|x|^{\frac{2-k}{2}}}{\tau^{\left(\frac{2 n}{2-k}+2\right)^{-1}}}\right)^{\frac{2 n}{2-k}+2}\right)^{p_{1}}, \\
& v_{A}(t, x) \approx c_{4}(T+t)^{\frac{1}{1-\beta_{2}}}\left(a+\left(\frac{|x|^{\frac{2-k}{2}}}{\tau^{\left(\frac{2 n}{2-k}+2\right)^{-1}}}\right)^{\frac{2 n}{2-k}+2}\right)^{p_{2}},
\end{aligned}
$$

at $|x| \tau^{-\frac{1}{n+2-k}} \rightarrow+\infty$, where the constants $c_{3}, c_{4}, p_{1}, p_{2}$ are defined above. 


\section{Numerical analysis of solutions}

We note that due to the non-uniqueness of solutions, many different cases arise in the numerical study of problem (1)-(2). It is important to choose a proper initial approximation that preserves its nonlinearity properties. On the basis of the above qualitative studies, we produced numerical calculations. The numerical results show quick convergence of the iterative process to the solution of the Cauchy problem (1)-(2), due to the successful choice of the initial approximation. Below, some numerical experiment results for different numerical parameter values are presented.

Results of the numerical experiments and graphs show that the self-similar solutions are very appropriate approximations. Fig. 1 shows a compactly supported solution of the problem (1)-(2). Figs. 2 and 3 show the properties of the solution for problem (1)-(2), vanishing at infinity.
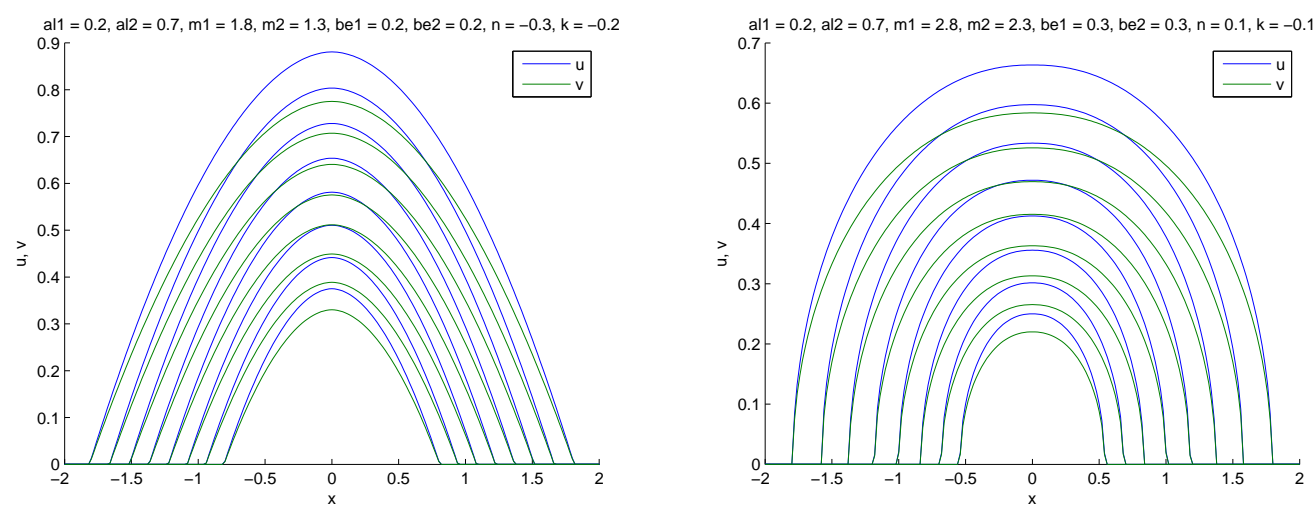

FIG. 1. Slow diffusion case $p_{i}>0, i=1,2: n<0, k<0$ and $n>0, k<0$
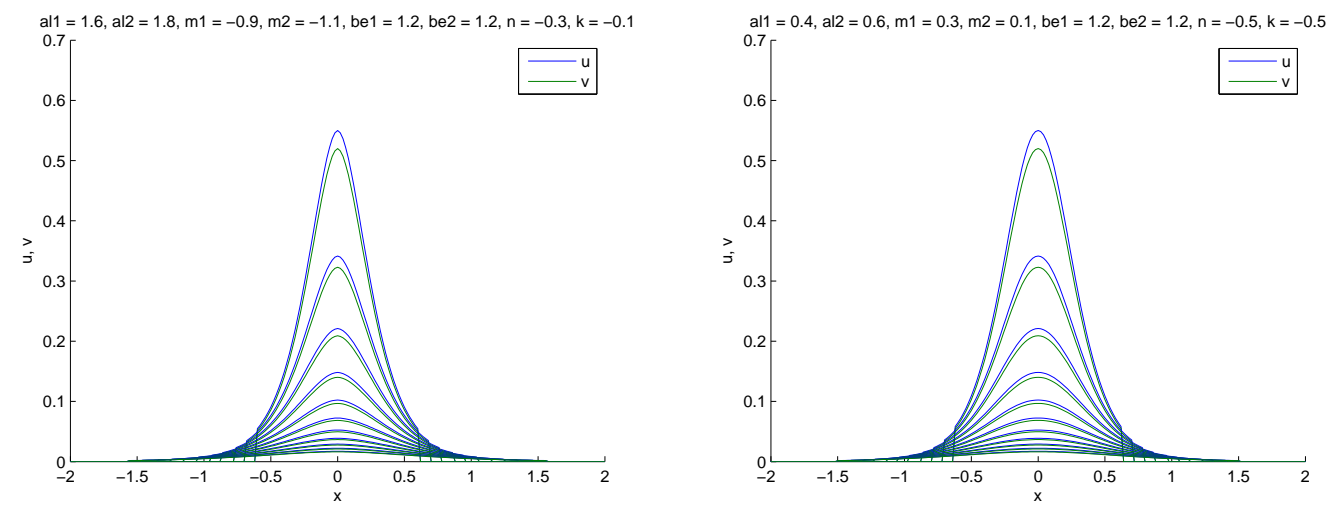

FIG. 2. Fast diffusion case $p_{i}<0, i=1,2: n-k<0$ and $n-k=0$

\section{Conclusions}

The Zeldovich-Barenblatt type solution for the cross diffusive system is obtained. The finite speed properties of the Cauchy problem for a cross-diffusion parabolic system with a source are studied using comparison method. The asymptotic behavior of self-similar solutions both for slow and fast diffusion cases are analyzed. It is shown that the coefficients in the main term of the asymptotic of the solution satisfy a system of nonlinear algebraic equations.

Results of the computational experiments show, that the self-similar solutions are very appropriate approximations and the iterative method based on the Picard method is effective for the numerical solution of nonlinear problems and leads to nonlinear effects if we use as initial approximation the solutions of self similar equations constructed by the nonlinear splitting and by the standard equation methods $[9,10,15]$. 

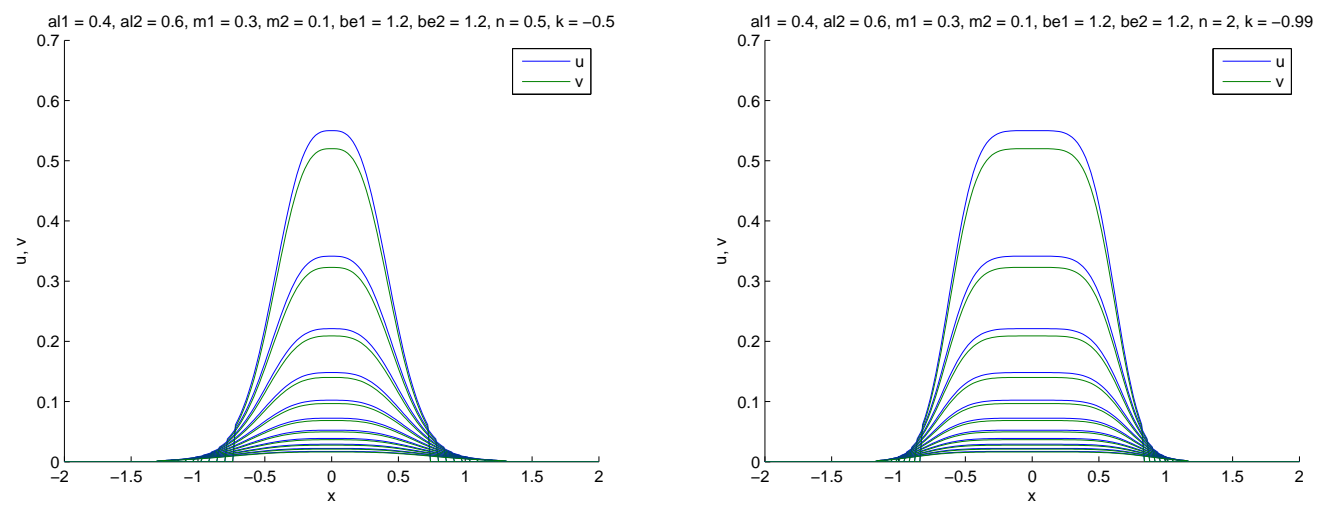

FIG. 3. Fast diffusion case $p_{i}<0, i=1,2: n-k=1$ and $n-k>1$

\section{References}

[1] Samarskii A.A., Galaktionov V.A., Kurdyumov S.P., Mikhailov A.P. Blow-up in Quasilinear Parabolic Equations. Walter de Grueter, Berlin, 1995, 4, $535 \mathrm{p}$.

[2] Wang M.X., Xie C.H. A degenerate strongly coupled quasilinear parabolic system not in divergence form. Z. Angew. Math. Phys., 2004, 55, P. 741-755.

[3] Haihua Lu. Global existence and blow-up analysis for some degenerate and quasilinear parabolic systems. Electronic Journal of Qualitative Theory of Differential Equations, 2009, 49, P. 1-14.

[4] Zhou W., Yao Z. Cauchy problem for a degenerate parabolic equation with non-divergence form. Acta Mathematica Scientia, 2010, 30 (5), P. 1679-1686.

[5] Chunhua J., Jingxue Y. Self-similar solutions for a class of non-divergence form equations. Nonlinear Differ. Equ. Appl. Nodea, 2013, 20 (3), P. 873-893

[6] Raimbekov J.R. The Properties of the Solutions for Cauchy Problem of Nonlinear Parabolic Equations in Non-Divergent Form with Density. Journal of Siberian Federal University. Mathematics and Physics, 2015, 8 (2), P. 192-200.

[7] Gao Y., Meng Q., Guo Y. Study of properties of solutions for quasilinear parabolic systems. MATEC Web of Conferences, 2016 , 61 (1), P. 1-4.

[8] Zhou Sh., Zheng S. Asymptotic behavior of solutions to a degenerate parabolic equation with a gradient source term. Asymptotic Analysis, 2015, 91 (2), P. 91-102

[9] Aripov M., Sadullaeva Sh.A. Qualitative Properties of Solutions of a Doubly Nonlinear Reaction-Diffusion System with a Source. Journal of Applied Mathematics and Physics, 2015, 3, P. 1090-1099.

[10] Aripov M., Sadullaeva Sh.A. An asymptotic analysis of a self-similar solution for the double nonlinear reaction-diffusion system. Nanosystems: physics, chemistry, mathematics, 2015, 6 (6), P. 1-10.

[11] Aripov M., Rakhmonov Z. Estimates and Asymptotic of Self-similar Solutions to a Nonlinear Filtration Problem with Variable Density and Nonlocal Boundary Conditions. Universal Journal of Computational Mathematics, 2016, 4 (1), P. 1-5.

[12] Sadullaeva Sh.A. Numerical Investigation of Solutions to A Reaction-Diffusion System with Variable Density. Journal of Siberian Federal University. Mathematics and Physics, 2016, 9 (1), P. 90-101.

[13] Martynenko A.V., Tedeev A.F. The Cauchy Problem for a Quasilinear Parabolic Equation with a Source and Inhomogeneous Density. Computational Mathematics and Mathematical Physics, 2007, 47, P. 238-248.

[14] Martynenko A.V., Shramenko V.N. Estimate of solutions of the Cauchy Problem near the Time of Exacerbation for a Quasilinear Parabolic Equation with a Source and a Variable Density. Nonlinear Boundary Value Problems, 2010, 20, P. 104-115.

[15] Aripov M. Standard Equation's Methods for Solutions to Nonlinear problems. Fan, Tashkent, 1988,138 p. 